\title{
REVIEWS
}

\section{Impact of HIV infection on tuberculosis}

\author{
Alimuddin Zumla, Patrick Malon, Jane Henderson, John M Grange
}

\begin{abstract}
Tuberculosis is increasing in prevalence in many countries and is now the leading infectious cause of death world wide, being responsible for three million deaths annually. Infection with HIV, likewise increasing in prevalence, has emerged as the most important predisposing factor for developing overt tuberculosis in people co-infected with Mycobacterium tuberculosis. Owing to the widespread geographical overlap of these two infections, it is estimated that in 1999, HIV related tuberculosis will reach one million cases and will cause $30 \%$ of the expected 2.5 million AIDS related deaths. Tuberculosis in HIV infected individuals may have unusual clinical features and can cause diagnostic difficulties. Despite the effectiveness of modern short course treatment, the mortality of HIV related tuberculosis during and after treatment remains high, and this may be due to other HIV related infections. The "cursed duet" of infection with both HIV and $M$ tuberculosis is generating a threat to human health of unparalleled proportions which, if not taken seriously by health workers and decision makers, could become totally unmanageable. (Postgrad Med f 2000;76:259-268)
\end{abstract}

Accepted 13 July 1999

Table 1 Historical names for tuberculosis

\begin{tabular}{ll}
\hline Historical name & Clinical type of tuberculosis \\
\hline Consumption (of the lungs) & Pulmonary tuberculosis \\
Pthisis & Pulmonary tuberculosis \\
Tabes pulmonalis & Pulmonary tuberculosis \\
Tissic & Pulmonary tuberculosis \\
Hectic fever & Pulmonary tuberculosis \\
Asthenia & Pulmonary tuberculosis \\
Galloping consumption & Acute progressive tuberculosis \\
Tabes mesenterica & Abdominal tuberculosis \\
Scrofula & Cervical lymphadenitis \\
Struma & Cervical lymphadenitis \\
King's evil & Cervical lymphadenitis \\
Pott's disease & Spinal tuberculosis \\
Prosector's or Butcher's wart & Primary inoculation skin tuberculosis \\
Tuberculous chancre & Skin tuberculosis \\
Scrofuloderma & Skin tuberculosis secondary to cervical lymphadenitis \\
Lupus vulgaris & Chronic skin tuberculosis
\end{tabular}

^Named after Sir Percival Pott (1714-88), a surgeon at St Bartholomew’s Hospital, London.

Table 2 Estimated global burden of tuberculosis (World Health Organisation, 1998)

\begin{tabular}{lllll}
\hline WHO region & Total population & No infected & Annual incidence $^{*}$ & Annual deaths \\
\hline South East Asia & 1458000000 & 704000000 & 2800000 & 1095000 \\
Western Pacific & 1630000000 & 610000000 & 1583000 & 591000 \\
Africa & 611000000 & 293000000 & 1650000 & 770000 \\
Americas & 788000000 & 237000000 & 448000 & 160000 \\
Europe & 859000000 & 205000000 & 342000 & 118000 \\
Eastern Mediterranean & 473000000 & 161000000 & 427000 & 173000 \\
Total & 5819000000 & 2210000000 & 7250000 & 2907000
\end{tabular}

${ }^{\star}$ Estimated number of new cases developing during the course of a year. Being a chronic disease, the total number of cases at a given time is much higher, around 16 million.
Keywords: HIV; AIDS; tuberculosis

Tuberculosis has been recognised since the dawn of recorded history and during the 19th century it was among the leading causes of morbidity and mortality in Europe and the USA. Some of many names that have been given to the various clinical forms of tuberculosis are listed in table 1. Starting in the latter half of the 19th century, the incidence declined significantly in the industrialised nations and by the early 1980s there was a widespread opinion that the disease had virtually been conquered. This complacent attitude was shaken in the early 1990 s by the occurrence of an upsurge in the incidence of tuberculosis in New York City. It is now apparent that, far from being conquered, tuberculosis is one of the most prevalent infectious causes of human suffering and death world wide. Indeed there are more cases of tuberculosis in the world today than at any previous time in human history. Because of the relentless spread of tuberculosis throughout the world, the World Health Organisation (WHO), in 1993, took the unprecedented step of declaring tuberculosis a global emergency. ${ }^{1}$

\section{Global burden of tuberculosis}

The global burden of tuberculosis is summarised in table 2. Each year, around seven to eight million people develop the disease. Tuberculosis causes the death of around three million people annually. The disease is responsible for $7 \%$ of all adult deaths and $25 \%$ of preventable adult deaths. ${ }^{2}$ Among children, it is now an important cause of morbidity and mortality. ${ }^{3}$ The incidence and prevalence of the disease continues to rise in the developing countries while many developed countries including the USA and UK have witnessed a reversal of the downward trend that had occurred since the late 19 th century.

\section{Aetiology of tuberculosis}

Most cases of human tuberculosis are caused by the human tubercle bacillus, Mycobacterium tuberculosis, but in countries where cattle tuberculosis still occurs human tuberculosis is also caused by $M$ bovis. In addition, some cases, principally in equatorial Africa, are caused by a rather heterogeneous group of strains termed $M$ africanum. Though bearing separate species names, these are really members of a single species often termed the $M$ tuberculosis complex. ${ }^{4}$ Infection usually occurs by inhaling small droplets of cough aerosol, about $5 \mu$ in diameter, containing tubercle bacilli. Infection 
Table 3 Factors determining susceptibility to tuberculosis

\begin{tabular}{ll}
\hline Age & $\begin{array}{l}\text { Those aged less than } 5 \text { years and the elderly are susceptible } \\
\text { The } 5-15 \text { age group is relatively protected } \\
\text { "Hacial and genetic predisposition } \\
\text { ? HLA }\end{array}$ \\
? ? Cytokine gene defects \\
HImmunological factors & $\begin{array}{l}\text { Steroid therapy } \\
\text { Cytotoxic drugs }\end{array}$ \\
& Post-transplant immunosuppressive therapy \\
& Congenital immunodeficiencies \\
& Protein energy malnutrition \\
Chronic medical conditions & Diabetes mellitus \\
& Chronic liver or renal disease \\
& Malignancies \\
Stress & Lung damage, especially silicosis \\
Previous exposure to mycobacteria & Endocrine factors \\
& BCG vaccination \\
Mycobacterial factors & Exposure to environmental mycobacteria \\
& Variation in strain virulence (?) \\
& Infective dose
\end{tabular}

Table 4 Global burden of HIVIAIDS, December 1998

\begin{tabular}{lll}
\hline Region & Persons infected with HIV & $\begin{array}{l}\text { Prevalence (\%) in } \\
\text { 15-49 age range }\end{array}$ \\
\hline Sub-Saharan Africa & 22500000 & 8.0 \\
North Africa and Middle East & 210000 & 0.13 \\
South and South East Asia & 6700000 & 0.69 \\
East Asia and Pacific & 560000 & 0.07 \\
Latin America & 1400000 & 0.57 \\
Caribbean & 330000 & 1.96 \\
Eastern Europe and Central Asia & 270000 & 0.14 \\
Western Europe & 500000 & 0.25 \\
North America & 890000 & 0.56 \\
Australia and New Zealand & 12000 & 0.1 \\
Total & 33400000 & 1.1 \\
\hline
\end{tabular}

by $M$ bovis is also acquired by drinking infected milk and the primary lesions occur in the pharynx or intestine. Rarely, infection occurs by traumatic inoculation of bacilli into the skin, particularly in butchers and others handling the carcasses of tuberculous animals.

\section{Factors determining susceptibility to} tuberculosis

Not everyone who is infected with the tubercle bacillus develops clinically evident disease. As a broad generalisation, and in the absence of obvious predisposing factors, about $5 \%$ of those infected develop primary tuberculosis within three years of infection and another 5\% develop post-primary tuberculosis at any subsequent period of their lives. ${ }^{5}$ Many factors predispose to the development of tuberculosis and the principal ones are listed in table 3 . The question of whether resistance to tuberculosis is genetically determined has generated considerable controversy. While various resistance determining genes have been delineated in the mouse, their relevance to the human situation has not been clearly established. Likewise, there is uncertainty as to whether the decline in the incidence of the disease in the developed nations over the last century is principally due to natural selection or to improving societal conditions.

Environmental factors may also affect susceptibility to progression to tuberculous disease. Thus, for example, it has been suggested that exposure to environmental mycobacteria may, depending on the nature of the exposure, have a beneficial or detrimental effect on the relevant immune responses. ${ }^{6}$ Age also affects susceptibility: the elderly and the very young appear more susceptible while children between the ages of 5 years and 15 years appear relatively immune from developing tuberculosis-the "golden age group". This age related variation in protection may be related to hormonal regulation of immune responses. $^{7}$

Impact of the HIVIAIDS pandemic on the global burden of tuberculosis

In addition to the factors mentioned above, immunosuppression of any type is a predisposing factor for the development of tuberculosis. Since the early 1980s, HIV infection has emerged as by far the most important of all the predisposing factors. ${ }^{8}$ In persons co-infected with the tubercle bacillus and HIV, the overall annual risk of developing active tuberculosis rises from about $0.4 \%$ to $8 \%$-that is, 20 times the risk. ${ }^{9}$ The risk depends, however, on the degree of immunosuppression-the risk of a patient with AIDS developing tuberculosis is 170 times higher than a nonimmunosuppressed person. For these reasons, infection with HIV and $M$ tuberculosis have been dubbed "the cursed duet". ${ }^{10}$

At the end of 1998, over 33 million people were estimated to be infected by $\mathrm{HIV}^{11}$ and the regional distribution of these is shown in table 4. Most of those infected were aged 15 to 49 years and, as shown in table 4 , over $1 \%$ of this age group are HIV positive. Assuming that a third of these are co-infected with the tubercle bacillus, and that $8 \%$ of these develop active tuberculosis annually, there could be almost one million cases of HIV related tuberculosis in 1999 , about $10 \%$ of the expected new cases of tuberculosis in that year. It has been estimated that tuberculosis will be the cause of $30 \%$ of the expected 2.5 million AIDS related deaths in $1999 .^{11}$

In addition to the high risk of developing reactivation tuberculosis, HIV positive persons can be primarily infected or reinfected by the tubercle bacillus and are at a very high risk of developing active tuberculosis. ${ }^{12}$ The risk of such infection and reinfection tuberculosis is related to the number of source cases in the community and is therefore low in regions with a low prevalence of tuberculosis. ${ }^{13}$ The exact risk of tuberculosis developing after exposure of an HIV positive person to a source case is unknown owing to the difficulty in distinguishing between recent infection and reactivation. Most documented cases follow exposure of patients to source cases during hospitalisation for AIDS related illness. These patients, by implication, are profoundly immunosuppressed and their chance of progressing to overt tuberculosis after infection approaches $100 \%$. Furthermore, the clinical course of the disease is "telescoped" down to a few months rather than years or decades. ${ }^{10}$ Such exposure in hospitals and other institutes has been responsible for a number of explosive miniepidemics, initially in New York City but subsequently in Europe. ${ }^{14}$ 
Prevalence and distribution of HIV related tuberculosis

The burden of HIV related tuberculosis is not evenly distributed as it is determined by the overall prevalence of infection by the tubercle bacillus and HIV in the community, in addition to the social factors that encourage a crossover between the two infections. In the USA, HIV related tuberculosis is largely restricted to socioeconomically disadvantaged communities in New York City and other large cities. ${ }^{15}$ It was the occurrence of miniepidemics of HIV associated tuberculosis in New York City in the early 1990s, and the anxieties generated in the general population, that led to an upsurge of interest and concern for this condition. In the UK, HIV related tuberculosis, while not yet a major problem, is becoming increasingly common in both immigrant and indigenous populations. Tuberculosis clinics are therefore encouraged to increase the use of HIV testing ${ }^{16}$ and HIV clinics should always keep tuberculosis high on the list of differential diagnosis in any difficult clinical case.

From the global perspective, the region most affected at the close of the millennium is sub-Saharan Africa, where $83 \%$ of all AIDS related deaths have occurred. ${ }^{17-19}$ However, the incidence of HIV infection is rapidly increasing in Asia, where a large percentage of the human population infected by the tubercle bacillus live. ${ }^{17}$ Within sub-Saharan Africa, East and Central African countries are particularly affected, with around one in five adults being infected with HIV. ${ }^{11}$ In these countries HIV is principally transmitted heterosexually, and infection in women is as frequent as men. In Zambia at least one in four pregnant women are HIV seropositive ${ }^{20}$ and, as around $50 \%$ are also infected with the tubercle bacillus, one in eight are likely to be co-infected. A recent study from Zambia indicates that HIV related tuberculosis is, after malaria, now the most important non-obstetric cause of maternal death. ${ }^{21}$ An increasing number of cases of tuberculosis in African children are HIV related. In Zambia, the HIV seroprevalence rate among children admitted to hospital with tuberculosis rose from $18 \%$ to $67 \%$ over an eight year period up to 1995 while over the same period, the HIV seroprevalence rate remained at a constant $10 \%$ in children admitted for surgical conditions. $^{22}$

\section{Pathology of tuberculosis in HIV infection}

The lungs of HIV positive patients dying with tuberculosis are characterised by fibrous and calcified tuberculosis lesions interspersed with newer active lesions containing tubercle bacilli. ${ }^{23}$ The latter may be due to either reactivation of old lesions or to reinfection. The precise nature of the immune deficit that leads to an enhanced risk of tuberculosis in HIV positive patients has not been established and a range of pathological characteristics have been described. In those with relatively intact immune function and a relatively high CD4+ count, there are classical caseating granulomas characterised by mature epithelioid cells and
Table 5 Comparison of clinical features of tuberculosis in $H I V$ infected and non-infected patients

\begin{tabular}{lll}
\hline Feature & $\begin{array}{l}\text { HIV } \\
\text { positive }\end{array}$ & $\begin{array}{l}\text { HIV } \\
\text { negative }\end{array}$ \\
\hline Respiratory symptoms & +++ & +++ \\
Extrapulmonary disease & +++ & + \\
Cavitating lung lesions & + & +++ \\
Atypical features on chest radiography & +++ & \pm \\
Negative tuberculin skin tests & ++ & \pm \\
Adverse drug reactions & ++ & \pm \\
Mortality rate & +++ & \pm \\
Relapse after course of treatment & ++ & \pm \\
\hline
\end{tabular}

Langhans' giant cells but few or no visible bacilli. As the immune defect increases, and the CD4+ cell count declines, more diffuse lesions with abundant tissue necrosis, few or no mature epithelioid cells and numerous tubercle bacilli are encountered. ${ }^{24}{ }^{25}$ Fibrotic reactions responsible for the walling off of foci of active disease are reduced in the more anergic patients, thereby encouraging local spread and widespread dissemination of disease. Cavity formation, also the result of an active, though inappropriate, immune response, is also reduced.

\section{Clinical features of HIV related tuberculosis}

The diagnosis of tuberculosis has never been easy as there is a very wide range of clinical features. The advent of HIV related tuberculosis, with many unusual presenting features, has added significantly to the diagnostic difficulties. Heightened clinical awareness remains the mainstay of diagnosis. Tuberculosis may develop at any point in the course of the HIV infection and may thus occur early in the course of HIV infection, often before any significant drop in CD4+ $\mathrm{T}$ cell counts occur or other clinical conditions suggestive of HIV infection or AIDS appear.

The nature, presentation, and the clinical and radiological features of tuberculosis depend on the degree of immunosuppression. In those with relatively good immunity and CD4+ cell counts, and a low viral load, the manifestations and presenting symptoms of tuberculosis are essentially similar to those in HIV negative persons. As the immunocompetence decreases, there is an increasing incidence of atypical presentations of tuberculosis, and diagnostic difficulties are posed by the rather non-specific presenting features which may be confused with those of other HIV related infections. ${ }^{3} 2627$ These atypical forms of tuberculosis include rapid progression of clinical disease after infection and a high proportion of extrapulmonary, multisite, and widely disseminated tuberculosis. The clinical features of HIV related and non-related tuberculosis are contrasted in table 5.

The most common extrapulmonary manifestations of tuberculosis are: (a) asymmetrical lymphadenopathy, (b) pericarditis, (c) pleurisy, and (d) bone and skin. For reasons that are not clear, involvement of the central nervous and genitourinary systems is, relative to the industrialised countries, uncommon in Africa. ${ }^{28}$ More generalised dissemination of disease may result in numerous minute lesions throughout 
the body. This condition, termed cryptogenic disseminated tuberculosis, may be very difficult to diagnose during life. Many unsuspected cases are therefore diagnosed at necropsy and pose serious risks to the pathologist. Tuberculin tests may be negative, particularly in the more severely immunosuppressed, although the extent of induration is not closely related to the CD4+ lymphocyte count and is thus an independent marker of immune competence. ${ }^{29}$ Blood cultures are positive in between a third and a half of patients with disseminated HIV related tuberculosis. ${ }^{30}$

A high proportion of African patients with AIDS develop severe wasting and is thus known locally as "slim disease". Many such patients have chronic diarrhoea and the condition was thus thought to be caused by enteropathy but in one study in Africa almost half the AIDS patients died with "slim disease" and almost half of these were found to have disseminated tuberculosis on necropsy, compared with just over a quarter of those dying without such wasting. ${ }^{31}$

\section{HIV related tuberculosis in children}

The clinical differences in the disease between HIV positive and HIV negative children are not as striking as in adults. ${ }^{22}$ In Africa, a large proportion of paediatric clinic attendances and hospital admissions are for pulmonary diseases and distinguishing tuberculosis from the other causes is never easy and is usually based on clinical features, tuberculin testing, and a history of exposure to a source case. Laboratory investigations are often unhelpful as lesions are usually closed and sputum, even when it can be obtained, is almost always negative for acid-fast bacilli on microscopy. Even when the most advanced diagnostic facilities are available, the diagnosis can only be confirmed by culture in about half the cases. ${ }^{32}$ Positive cultures may be obtained from unusual sites: in a study in South Africa six of 14 HIV infected children with culture positive tuberculosis had otorrhoea and ear swabs were the source of the positive cultures in three such cases. ${ }^{33}$

Additional diagnostic difficulties are experienced in HIV positive children as the common diagnostic criteria such as chronic cough, weight loss, and failure to thrive may also be the result of other HIV related pulmonary infections such as Pneumocystis carinii pneumonia and disease due to environmental mycobacteria, especially members of the $M$ avium complex. ${ }^{34}$ Likewise, the radiological characteristics of these other infections may be indistinguishable from those of tuberculosis. Thus, in the developing countries, misdiagnosis is common, rendering it difficult to assess the magnitude of the problem of HIV related tuberculosis in children. An idea of the true risk of tuberculosis in HIV infected children may be obtained from necropsy studies. One such study in West Africa suggested a low risk ${ }^{35}$ but necropsies performed on HIV positive children in Bulawayo, Zimbabwe, established a diagnosis of tuberculosis in six out of 122 children $(5 \%) .{ }^{36}$ A large necropsy study of children dying from respiratory diseases is currently underway in Zambia.

\section{Diagnosis of tuberculosis}

In view of the problems encountered in the clinical diagnosis of tuberculosis, a huge amount of effort has been placed in the development of rapid and sensitive diagnostic tests but serious problems have been encountered. Traditionally, diagnosis has been made by the microscopical demonstration of acid-fast bacilli in biological specimens and by in vitro cultivation of tubercle bacilli. Neither approach is completely satisfactory and attention has recently turned to molecular methods of diagnosis.

\section{MICROSCOPY}

This is principally applied to sputum but other specimens include bronchoalveolar lavage fluid, gastric washings, laryngeal swabs, cerebrospinal fluid, pleural, pericardial and peritoneal effusions, fine needle lymph node aspirates, bone marrow aspirates, and tissue biopsies. Ideally, three sputum specimens collected on successive days should be examined but, particularly in resource poor countries, this encourages non-compliance and overburdens the laboratory. ${ }^{4}$ Children rarely produce sputum: only $5 \%$ of cases of childhood pulmonary tuberculosis are smear positive. Gastric aspirates are more likely to be positive but laryngeal swabbing is distressing to the child, exposes the operator to risk of infection, and gives a low diagnostic yield.

Microscopy of sputum is rapid, permitting a presumptive diagnosis to be made on the patient's first visit to the clinic, but it is insensitive as there must be at least 5000 organisms in $1 \mathrm{ml}$ of sputum to render their detection likely. Microscopy does not permit the distinction between tubercle bacilli and environmental mycobacteria, but this is not a major problem in regions where tuberculosis is common. As mentioned above, patients with HIV related tuberculosis, particularly those with more profound immunosuppression and no cavity formation, are more likely to be sputum negative than those with typical cavitating postprimary disease.

\section{CULTURE}

By facilitating an identification at species level, isolation of $M$ tuberculosis in culture provides a definitive diagnosis. The traditional method of inoculating solid media such as the egg based Löwenstein-Jensen medium is sensitive but slow as growth may not be visible until after three or more weeks' incubation. More rapid results are obtained by the use of commercially available culture systems based on the liberation of radiolabelled carbon dioxide $\left(\mathrm{CO}_{2}\right)$ or changes in the colour or fluorescence of dyes due to the liberation of $\mathrm{CO}_{2}$ or consumption of oxygen. ${ }^{4}$

SEROLOGICAL TESTS

There have been numerous attempts to develop serological tests for tuberculosis and a few are commercially available but serious 
problems of specificity and sensitivity have been encountered. Owing to compromised immune responses, sensitivity is even lower in patients with HIV related tuberculosis. ${ }^{37}$

MOLECULAR METHODS

In view of the problems encountered with the traditional microbiological methods, the application of nucleic acid (DNA and RNA) amplification techniques - the polymerase chain reaction (PCR) and the ligase chain reaction (LCR) - is the subject of intense research activity. As a result, a number of rapid, sensitive, and specific test kits are commercially available. ${ }^{38}$ Problems arise as a result of the presence of inhibiting substances in some clinical specimens which reduce sensitivity, especially in smear negative sputum specimens, and the unexplained occurrence of a few "false" positive results. ${ }^{4}$ Another problem is cross contamination, although this is minimised by closed, isothermal, systems based on the amplification of specific messenger RNA which has a short half life.

Molecular techniques also permit the rapid identification of mycobacteria other than $M$ tuberculosis and the determination of rifampicin resistance by detection of mutations in the $R p o B$ gene responsible for resistance. ${ }^{39}$

A further application of molecular technology is the "fingerprinting" of isolates, or PCR products, of $M$ tuberculosis. Such techniques have been used to investigate miniepidemics of HIV related tuberculosis and to differentiate between reactivation of old lesions and recent infection. It has thus been shown that, particularly among HIV positive persons, reinfection occurs much more frequently than previously expected. ${ }^{40}$ At the present time, the cost of nucleic based technology rather than technical problems precludes its use in resource poor countries.

\section{CHEST RADIOGRAPHY}

The radiological appearance of pulmonary tuberculosis in both HIV negative and positive is very variable, but more so in the latter. ${ }^{41}{ }^{42}$ Classical chest radiograph appearances of tuberculosis are seen in approximately one third of HIV infected patients while the others show a range of atypical appearances. Radiological studies of adult Zairean and Zambian patients with HIV related tuberculosis show a significantly increased incidence of lymphadenopathy, pleural effusions, parenchymal changes, consolidation and miliary disease, but significantly less cavitary disease and atelectasis. ${ }^{42}$ Intrathoracic lymphadenopathy, uncommon in HIV uninfected adults with post-primary tuberculosis, is evident in $25 \%$ $50 \%$ in HIV infected adults with pulmonary tuberculosis. ${ }^{43}$ The factors determining the radiological appearance of tuberculosis in HIV positive patients are poorly understood. Contrary to previous opinion, the ability to form cavities and fibrotic changes do not appear to be directly related to the CD4+ cell counts. The occurrence of atypical radiological manifestations of HIV related pulmonary tuberculosis has implications for the accurate diagnosis of the disease and a high degree of clinical suspicion is therefore required.

Treatment of HIV related tuberculosis

The standard WHO recommended antituberculosis regimen is a six month course of rifampicin and isoniazid, with the addition of pyrazinamide, together with ethambutol (or streptomycin) during the first two months of treatment. Supplementation with daily pyridoxine (vitamin B6) to prevent isoniazid induced neuropathy is now routine.

Antituberculous regimens based on drugs other than rifampicin, or the use of rifampicin only during the first two months, have been used on the grounds that they are cheaper. The cost difference is now not so great and, in terms of relapse rates and duration of treatment, the rifampicin based regimens are ultimately much more cost effective.

Thiacetazone based regimens, though cheap, cause an unacceptably high incidence of severe toxic reactions in HIV infected persons and they should be universally abandoned.

There is little or no difference in relapse rate between HIV infected and uninfected patients when rifampicin based short course treatment is used. Thus, in the absence of drug resistance, the standard short course described above is recommended. ${ }^{44}$ Nevertheless, some physicians continue treatment for nine months to further reduce the risk of relapse. ${ }^{45}{ }^{46}$ Unfortunately, as outlined below, many patients die of other HIV related complications during or after completion of antituberculosis treatment. In one study, extending rifampicin based treatment from six to 12 months reduced the relapse rate but did not improve survival. ${ }^{47}$

In addition, owing to a range of human factors, including negative perception of the disease, its treatment and outcome and the stigmatising nature of the diagnosis, HIV positive patients in some regions are less likely than seronegative patients to complete treatment. ${ }^{48}$ Thus it is essential to adopt strategies of directly observed therapy (DOT) in order to ensure completion of treatment. ${ }^{44}$ This strategy may also help to limit the emergence of drug resistant tuberculosis but, in several African countries, even the establishment of national tuberculosis programmes employing DOT based strategies, is failing to stem the rising tide of tuberculosis. In Botswana, for example, the incidence of tuberculosis rose by $120 \%$ between 1989 and 1996, paralleling that of the prevalence of HIV infection, despite a decade of such strategies and a low prevalence of drug resistance. $^{49}$

\section{Adverse effects of antituberculosis chemotherapy}

Adverse effects of first line antituberculosis drugs occur in both HIV infected and uninfected patients and the patient should be monitored carefully for these. Side effects of antituberculosis drugs are comparatively more frequent in the HIV infected tuberculosis patients and very severe and even fatal reactions have been observed in several studies. The most serious problems are encountered 
Table 6 Drug interactions with rifampicin *

\begin{tabular}{ll}
\hline Effect of drug opposed by rifampicin & \\
Protease inhibitors & Non-nucleoside reverse transcriptase inhibitors \\
Azathioprine & Cyclosporin \\
Diazepam & Digoxin \\
Glucocorticoids & Haloperidol \\
Imidazole antifungals & Oral contraceptives \\
Opiods & Phenytoin \\
Propanolol & Quinidine \\
Theophylline & Tolbutamide \\
Warfarin & Cotrimoxazole \\
Effects of rifampicin potentiated &
\end{tabular}

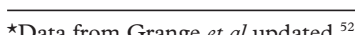

with thiacetazone, notably dermal reactions including exfoliative dermatitis and the frequently fatal Stevens-Johnson syndrome. In one study of adults from Kenya, thiacetazone toxicity was 18 times more frequent in HIV positive, than in HIV negative, patients and the risk was directly related to the degree of immunosuppression, suggesting an immunological basis. ${ }^{50}$ A study of Zambian children demonstrated severe fatal reactions to thiacetazone and highlighted the advantages of thiacetazone-free regimens. ${ }^{51}$ Accordingly, as mentioned above, regimens containing thiacetazone should no longer be used.

\section{Antituberculous drug interactions}

A number of interactions between antituberculosis agents and other drugs have been described. ${ }^{52}$ Most interactions are associated with rifampicin due to its ability to induce cytochrome CYP450 enzymes in the liver which affect the metabolism of many other drugs (table 6). In addition to affecting the metabolism of antifungals and other drugs that HIV positive patients may require, serious interactions may occur between rifampicin and antiretroviral drugs (protease inhibitors and non-nucleoside reverse transcriptase inhibitors). The recommended practice was to stop antiretroviral treatment so that rifampicin could be used to treat tuberculosis but the current recommendation is to replace rifampicin by rifabutin, a much less powerful inducer of cytochrome enzymes, and to start or continue with the antiretroviral drugs. ${ }^{46}$ Even if rifabutin is substituted for rifampicin, care should be exercised in the use of the protease inhibitor saquinavir and the non-nucleoside reverse transcriptase inhibitors as rifabutin decreases their levels, with the risk of selection of drug resistant viruses. ${ }^{8}$ Another problem encountered with the simultaneous administration of antiretrovirals and antituberculosis chemotherapy is the temporary exacerbation of the symptoms and signs of tuberculosis-the so-called paradoxical reactions..$^{53}$ These, which have been ascribed to hypersensitivity reactions to antigen released by bacilli killed by the chemotherapy, manifest as fever, enlargement of affected lymph nodes, and a worsening of the radiological appearance. They are occasionally encountered in HIV seronegative patients but their incidence is higher in HIV seropositive patients, particularly those given antiretroviral drugs, probably as a result of improving immune responsiveness. These reactions are not indicative of treatment failure and usually subside spontaneously. Treatment should not be modified but short courses of steroids may be required for severe paradoxical reactions. ${ }^{46}$

\section{Mortality in patients with HIV related tuberculosis}

There is considerable evidence that HIV seropositive patients with tuberculosis are at a higher risk of dying than their HIV seronegative counterparts during or after treatment for tuberculosis, with death usually being caused by complications of HIV infection rather than to tuberculosis itself. ${ }^{54}$ A study in Malawi showed the former were 2.5 times as likely to die during treatment, with half the deaths occurring during the first month of treatment. ${ }^{56}$ The risks of dying for those with smear negative and extrapulmonary tuberculosis were, respectively, 3.9 and 2.6 higher than those with sputum positive disease, probably as the former tend to be associated with more profound immunosuppression. The risk of death is related to the antituberculosis regimen used. In Uganda, the risk of death within a year of starting treatment was $60 \%$ higher in patients treated with streptomycin, thiacetazone, and isoniazid than in those receiving rifampicin-based regimens. ${ }^{57}$ This may be attributable to the prevention of other opportunistic AIDS related infections by the broad antimicrobial activity of rifampicin. Nevertheless, it is important to diagnose and treat other opportunistic infections that occur during the course of antituberculosis treatment. ${ }^{44}$

The reason for the high mortality associated with the onset of HIV related tuberculosis, even when treated vigorously, is not clear. It may be the result of a poorly understood synergy between the two infections which accelerates the decline in immune competence and also greatly increases the viral load. ${ }^{58}$ This synergy may be due to an induction of HIV replication by cytokines, including tumour necrosis factor- $\alpha$, induced by the tuberculous process. In addition, tuberculosis per se is associated with a reduction in the CD4+ lymphocyte count which may be additive to that resulting from the HIV infection. In view of the deleterious effect of tuberculosis on the course of HIV infection, all forms of tuberculosis are classified as AIDS defining conditions. ${ }^{59}$

\section{Drug resistant tuberculosis}

A further complicating factor is the occurrence of drug resistant tuberculosis. Many of the cases in these outbreaks reported in New York City were multidrug resistant which, by definition, are resistant to rifampicin and isoniazid with or without resistance to other drugs. ${ }^{60}$ This was the result of poor medical care previously received by the patients who were mostly from socioeconomically underprivileged sectors of the community. HIV infection per se is not a predisposing factor for the development of multidrug resistance and, in many other parts of the world, such an association does not occur. ${ }^{6162}$ Tests for drug susceptibility are often not possible in developing countries but they 
should certainly be performed in the industrialised nations. ${ }^{44}$

Infection control in health care settings As several miniepidemics of HIV related tuberculosis, including multidrug resistant forms, have followed exposure to source cases within hospitals and clinics, well defined policies for the prevention of transmission of tuberculosis within such institutions, including isolation of known and suspect infectious patients, are essential. $^{63}$

\section{Prophylaxis against tuberculosis in co-infected persons}

In view of the very high risk of a co-infected person developing active tuberculosis, and the adverse effect of this disease on the immune status and survival of the patient, there is a very good theoretical case for provision of prophylactic treatment for those at risk. In practice, serious problems have been encountered in diagnosing dual infection, ruling out active tuberculosis, and ensuring compliance with treatment without breach of confidence or enhancement of stigma. ${ }^{6364}$

In the initial studies, a 12 month course of isoniazid was found to lower the incidence of tuberculosis in co-infected persons. ${ }^{65}{ }^{66}$ Subsequently, shorter combination regimens have also been shown to be effective. These include a three month course of a rifamycin (rifampicin or rifabutin) plus isoniazid and a two month course of a rifamycin plus pyrazinamide. ${ }^{46}{ }^{67-69}$ A study in Zambia revealed that the two month combination regimens or six months of isoniazid, administered twice weekly, reduced the incidence of tuberculosis by about $40 \%$ compared with a placebo group, although the overall mortality due to all causes was not reduced. ${ }^{70}$ Prevention was more effective in those with relatively limited immunosuppression (positive tuberculin tests, high lymphocyte counts, and high haemoglobin concentrations). By 18 months, the incidence of tuberculosis in those who had received prophylaxis was similar to that in the placebo group; probably due to reinfection and thus indicating the need to consider repeated courses or, perhaps, lifelong prophylactic treatment.

\section{HIV infection and tuberculosis of bovine origin}

Tuberculosis due to $M$ bovis is transmitted from cattle to humans and, occasionally from humans to cattle, but the evidence for human to human transmission is weak and anecdotal. ${ }^{71}$ This has led to the concept, never formally proved, that $M$ bovis is less virulent for humans than $M$ tuberculosis. There have, however, been reports of a high incidence of HIV related tuberculosis due to $M$ bovis among Mexicans arriving in the $\mathrm{USA}^{72}$ and also a report of a miniepidemic resulting from exposure in an AIDS unit to a patient infected with a tubercle bacillus closely resembling $M$ bovis. ${ }^{14}$ If confirmed, an increased susceptibility of HIV infected persons to this form of tuberculosis could generate a serious human health prob- lem in regions, including many African countries, where the prevalence of HIV infection is high and where cattle tuberculosis has not been eradicated. $^{73}$

BCG vaccination in HIV infected persons There is a risk that vaccination with Bacille Calmette-Guérin (BCG), a living attenuated vaccine, will cause infectious complications in HIV positive persons. ${ }^{74}$ There is a small increase in the incidence of adverse effects of BCG in the children of HIV infected women, but most such effects are mild. ${ }^{75}$ In one study, complications occurred in nine of $68 \mathrm{HIV}$ infected children three to 35 months after neonatal BCG vaccination. Regional lymphadenopathy with or without fistula formation occurred in seven and systemic disease in two. ${ }^{76}$ Accordingly, the safety of BCG vaccination in regions with a high incidence of HIV infection requires consideration. The WHO has advised that, while persons known to be HIV positive should never be given BCG, routine immunisation of infants should nevertheless continue in areas with a high incidence of tuberculosis and HIV infection. ${ }^{77}$ In the UK it is recommended infants born to mothers known or suspected to be HIV positive that should not receive BCG unless they are subsequently shown to be HIV negative. ${ }^{78}$

\section{Conclusions}

The emergence of the HIV pandemic over the last two decades has had a devastating effect on tuberculosis world wide, but principally in subSaharan Africa. In 1999, around 10\% of all cases of tuberculosis will be HIV related, rising to over $20 \%$ in Africa. In addition, $30 \%$ of the expected 2.5 million AIDS related deaths in 1999 will be due to tuberculosis. It is expected that the problem will likewise become extensive in Asia over the next decade. Many African countries report that their health care facilities are overwhelmed by the additional burden of HIV related tuberculosis and urgent action is therefore required to stem this epidemic before it becomes totally unmanageable, with devastating human and economic consequences which will be felt throughout the world.

The difficulties in diagnosis posed by the varied clinical manifestations of tuberculosis and the particularly high incidence of atypical presentations in HIV positive patients, together with the problems of management, must be borne in mind by the practising physician. As the incidence of tuberculosis, especially of cases that are HIV related, continues to escalate world wide, physicians and pathologists must keep tuberculosis high on the list of differential diagnoses in any problematic clinical case.

\footnotetext{
1 World Health Organisation. TB-a global emergency. Geneva: WHO, 1994.

2 Murray CJ, Styblo K, Rouillon A. Tuberculosis in developing countries: burden, intervention and cost. Bulletin of the International Union of Tuberculosis and Lung Disease 1990;65: 6-24.

3 Chintu C, Zumla A. Pediatric tuberculosis and the HIV epidemic. In: Zumla A, Johnson M, Miller RF, eds. AIDS and demic. In: Zumla A, Johnson M, Miller RF, eds. AIDS and
respiratory medicine. London: Chapman and Hall, 1997: respiratory
} 
4 Collins CH, Grange JM, Yates MD. Tuberculosis bacteriology. Organization and practice. 2nd Ed. Oxford: Butterworth Heinemann, 1997.

5 Raviglione MC, Snider DE, Kochi A. Global epidemiology of tuberculosis. Morbidity and mortality of a worldwide epidemic. $\mathcal{F} A M A$ 1995;273:220-6.

6 Fine PEM. Variation in protection by BCG: implications of and for heterologous immunity. Lancet 1995;346:1339-45.

7 Rook GAW, Hernandez-Pando R, Baker R, et al. Human and murine tuberculosis as models for immuno-endocrine
interactions. In: Rook GAW, Lightman S, eds. Steroid hormones and the T cell cytokine profile. London: Springer,

8 Havlir DV, Barnes PE. Tuberculosis in patients with human mmunodeficiency virus infection. N Engl f Med 1999;340 367-73.

9 Dolin PJ, Raviglione MC, Kochi A. Global tuberculosis incidence and mortality during 1990-2000. Bull World Health Organ 1994;72:213-20.

10 Chretien J. Tuberculosis and HIV. The cursed duet. Bull Int Union Tuberc Lung Dis 1990;65:25-8.

11 UNAIDS. AIDS epidemic update, December 1998. Geneva: World Health Organisation, 1998.

12 Alland D, Kalkut GE, Moss AR, et al. Transmission of tuberculosis in New York City - an analysis by DNA fingerprinting and conventional epidemiologic methods. N Engl f Mrinting and conventiona $1994 ; 330: 1710-16$.

13 Leitch AG, Rubilar M, Watt B, et al. Why disease due to Mycobacterium tuberculosis is less common than expected in HIV-positive patients in Edinburgh. Respir Med 1995;89: 495-7.

14 Bouvet E, Casalino E, Mendoza-Sassi G, et al. A nosocomial outbreak of multidrug-resistant Mycobacterium bovis
among HIV-infected patients. A case-control study. AIDS 1993;7:1453-60.

15 Coker R. Lessons from New York's tuberculosis epidemic. BMF 1998;317:616.

16 Coker R, Miller R. HIV associated tuberculosis. BMF 1997; 314:1847.

17 World Health Organisation. The world health report 1998 Geneva: WHO, 1998.

18 Ustianowski A, Mwaba P, Zumla A. Tuberculosis and HIV-perspectives from sub-Saharan Africa. In: Porter $\mathrm{JDH}$, Grange JM, eds. Tuberculosis - an interdisciplinary perspective. London: Imperial College Press, 1999.

19 Blinkhoff P, Bukanga E, Syamalevwe B, et al. Under the mupundu tree. Strategies for Hope Series No 14. London: Actionaid, 1999

20 Fylkesnes K, Musonda RM, Kasumba K, et al. The HIV epidemic in Zambia: socio-demographic prevalence patterns and indications of trends among childbearing women. AIDS 1997;11:339-45.

21 Ahmed Y, Mwaba P, Grange JM, et al. A study of maternal mortality at the University Teaching Hospital, Lusaka, Zambia: the emergence of tuberculosis as a major non-obstetric cause of maternal death. International fournal of Tuberculosis and Lung Disease 1999;3:1-6.

22 Chintu C, Zumla A. Childhood tuberculosis and infection with the human immunodeficiency virus. F $R$ Coll Phys Lond 1995;29:92-94.

23 Lucas S, Nelson AM. Pathogenesis of tuberculosis in human immunodeficiency virus-infected people. In: Bloom BR, ed. Tuberculosis: pathogenesis, protection and control. Washington: ASM Press, 1994: 503-13.

24 Lucas S, Wamukota W. HIV and the local African population. In: Pounder RE, Chiodini PL, eds. Advanced medicine. London: Balliere-Tindall, 1987: 102-11.

25 Nambuya A, Sewankambo N, Mugerwa J, et al. Tuberculous lymphadenitis associated with human immunodeficiency
virus infection in Uganda. f Clin Pathol 1988;41:93-6.

26 Huebner RE, Castro KG. The changing face of tuberculosis. Ann Rev Med 1995;46:47-55.

27 Whalen C, Horsburgh CR, Hom D, et al. Accelerated course of human immunodeficiency virus infection after tuberculosis. Am $\mathcal{F}$ Resp Crit Care Med 1995;151:129-34

28 Harries AD. Tuberculosis in HIV-infected persons with special emphasis on sub-Saharan Africa. F Infect 1998;37:2059.

29 Diagbouga S, Fumoux F, Ledru E, et al. Lack of direct correlation between $\mathrm{CD} 4+$ lymphocyte counts and induration sizes of the tuberculin skin test in human immunodeficiency virus type 1 seropositive patients. International fournal of Tuberculosis and Lung Disease 1998;2:317-23.

30 Di Lonardo M. Isola NC, Ambroggi M, et al. Mycobacteria in HIV-infected patients in Buenos Aires. Tuber Lung Dis 1995;76:185-9.

31 Lucas SB, de Cock KM, Hounnou A, et al. Slim disease in Africa: the contribution of tubeculosis. BMF 1994;308: 1531-3.

32 Starke JR, Taylor-Watts RT. Tuberculosis in the paediatric population in Houston, Texas. Pediatrics 1989;84:28-35.

33 Schaaf HS, Geldenduys A, Gie RP, et al. Culture-positive tuberculosis in human immunodeficiency virus type 1-infected children. Pediatr Infect Dis f 1998;1 7:599-604.

34 Donald PR. Clinical manifestations of tuberculosis. In: Donald PR, Fourie B, Grange JM, eds. Tuberculosis in childhood. Pretoria: JL van Schaik, 1999: 61-102.

35 Lucas SB, Peacock CS, Hounnou A, et al. Disease in children infected

36 Ikeogu MO, Wolf B, Mathe S. Pulmonary manifestations in HIV seropositivity and malnutrition in Zimbabwe. Arch Dis HIV seropositivity and
Child $1997 ; 76: 124-8$.
37 Wilkins EGL. Antibody detection in tuberculosis. In: Davies PDO, ed. Clinical tuberculosis. London: Chapman and Hall, 1998: 81-96.

38 Tan MF, Ng WC, Chan SH, et al. Comparative usefulness of PCR in the detection of Mycobacterium tuberculosis indifferent clinical specimens. $\mathcal{F}$ Med Microbiol 1997;46:164-9.

39 Drobniewski FA, Wilson SM. The rapid diagnosis of isoniazid and rifampicin resistance in Mycobacterium tubercul

40 van Embden JDA, Cave MD, Crawford JT. Strain identification of Mycobacterium tuberculosis by DNA fingerprinting: recommendations for a standardised methodology. f Clin Microbiol 1993;31:406-9.

41 Daley CL. The typically "atypical" radiographic presentation of tuberculosis in advanced HIV disease. Tuber Lung Dis 1995:76:475-6.

42 Tshibwabwa-Tumba E, Mwinga A, Pobee JOM, et al. Radiological features of pulmonary tuberculosis in 963 HIV-infected adults at three central African hospitals. Clin Radiol 1997;52:837-41.

43 Saks AM, Posner R. Tuberculosis in HIV positive patients in South Africa: a comparative radiological study with HIV negative patients. Clin Radiol 1992;46:387-90.

44 Scientific Statement. Treatment regimens in HIV-infected tuberculosis patients. An official statement of the International Union Against Tuberculosis and Lung Disease. International fournal of Tuberculosis and Lung Disease 1998;2: Internation.

45 Murray JF. Tuberculosis and HIV infection: a global perspective. Respiration 1998;65:335-42.

46 Centers for Disease Control. Prevention and treatment of tuberculosis among patients infected with human immunodeficiency virus: principles of therapy and revised recommendations.

47 Perriens JH, St Louis ME, Mukudi MB, et al. Pulmonary tuberculosis in HIV-infected patients in Zaire: a controlled trial of treatment for either 6 or 12 months. $N$ Engl $\mathcal{F}$ Med 1995;32:779-84.

48 Ackah AN, Coulibaly D, Digbeu H, et al. Response to treatment, mortality, and CD4 lymphocyte counts in HIVment, mortality, and CD4 lymphocyte counts in HIVinfected persons with tuberc

49 Kenyon TA, Mwasekaga MJ, Huebner R, et al. Low levels of drug resistance amidst rapidly increasing tuberculosis and human immunodeficiency virus co-epidemics in Botswana. International fournal of Tuberculosis and Lung Disease 1999;3: 4-11.

50 Nunn P, Kibuga D, Gathua S, et al. Cutaneous hypersensitivity reactions due to thiacetazone in HIV-1 seropositive patients treated for tuberculosis. Lancet 1991;337:627-30.

51 Luo C, Chintu C, Bhatt G, et al. HIV-infection in Zambian children with tuberculosis: changing seroprevalence and evaluation of a thiacetazone-free regimen. Tuber Lung Dis 1994;75:111-15.

52 Grange JM, Winstanley PA, Davies PDO. Clinically significant drug interactions with antituberculosis agents. Drug Saf 1994;11:242-51.

53 Narita M, Ashkin D, Hollender ES, et al. Paradoxical worsening of tuberculosis following antiretroviral therapy in patients with AIDS. Am $\mathcal{F}$ Respir Crit Care Med 1998;158: 157-61.

54 Perriens JH, Colebunders RL, Karahunga C, et al. Increased mortality and tuberculosis treatment failure rate among human immunodeficiency virus (HIV) seropositive compared with HIV seronegative patients with pulmonary tuberculosis treated with "standard" chemotherapy in Kinshasa, Zaire. Am Rev Respir Dis 1991;144:750-5.

55 van den Broek J, Mfinanga S, Moshiro C, et al. Impact of human immunodeficiency virus on the outcome of treatment and survival of tuberculosis patients in Mwanza, Tanzania. International fournal of Tuberculosis and Lung Disease $1998 ; 2: 547-52$.

56 Harries AD, Nyangulu DS, Kang'ombe C, et al. Treatment outcome of an unselected cohort of tuberculosis patients in relation to human immunodeficiency virus status in Zomba Hospital, Malawi. Trans R Soc Trop Med Hyg 1998;92:343-

57 Okwera A, Whalen C, Bykewaso F, et al. Randomised trial of thiacetazone and rifampicin-containing regimens for pulmonary tuberculosis in HIV-infected Ugandans. Lancet 1994;334:1323-8.

58 Goletti D, Weissman D, Jackson RW, et al. Effect of Mycobacterium tuberculosis on HIV replication: role of immune activation. F Immunol 1996;157:1271-6.

59 Centers for Disease Control. The 1993 revised classification system for HIV infection and expanded surveillance case
definition for AIDS among adolescents and adults. $M M W R$ definition for AIDS among adolescents and
Morb Mortal Wkly Rep 1993;41(17):1-19.

60 Kochi A, Vareldzis B, Styblo K. Multidrug-resistant tuberculosis and its control. Res Microbiol 1993;144:104-10.

61 Gordin FM, Nelson ET, Matts JP, et al. The impact of human immunodeficiency virus infection on drug resistant 2 Mitike G, Kebede D, Yenneneh $\mathrm{H}$. HIV infection and antituberculosis drug resistance among pulmonary tuberculosis patients in Harar Tuberculosis Centre, Ethiopia. East Afr Med f 1997; 74:154-7.

63 International Union of Tuberculosis and Lung Disease/ International Union of Tuberculosis and Lung Disease/ World Health Organisation. Statement: tuberculosis preven-
tive therapy in HIV-infected individuals. Tuber Lung Dis 1994;75:96-8. 
64 O'Brien RJ, Perriens JH. Preventive therapy for tuberculosis in HIV infection: the promise and the reality. AIDS 1995;9: in HIV in

65 Pape JW, Jean SS, Ho JL, et al. Effect of isoniazid prophylaxis on incidence of active tuberculosis and progression of HIV infection. Lancet 1993;342:268-72.

66 Hawken MP, Meme HK, Elliott LC, et al. Isoniazid preventive therapy for tuberculosis in HIV-1 infected adults: results of a randomised controlled trial. AIDS 1997;11:875-82.

67 Wilkinson D, Squire SB, Garner P. Effect of preventive treatment for tuberculosis in adults infected with HIV: systematic review of randomised placebo controlled trials. $B M Y$ 1998;317:625-9.

68 Rose DN. Short-course prophylaxis against tuberculosis in HIV-infected persons. A decision and cost-effectiveness analysis. Ann Intern Med 1998;129:779-86.

69 Halsey NA, Coberly JS, Desormeaux J, et al. Randomised trial of isoniazid versus rifampicin and pyrazinamide for trial of isoniazid versus rifampicin and pyrazinamide for
prevention of tuberculosis in HIV-1 infection. Lancet 1998; prevention of

70 Mwinga A, Hosp M, Godfrey-Faussett P, et al. Twice weekly tuberculosis preventive threrapy in HIV infection in Zambia. AIDS 1998;12:2447-57

71 Grange JM, Collins CH. Tuberculosis and the cow. fournal of the Royal Society of Health 1997;117:119-22.

72 Dankner WM, Waecker NJ, Essey MA, et al. Mycobacterium bovis infections in San Diego: a clinicoepidemiological study of 73 patients and a historical review of a forgotten pathogen. Medicine 1993;72:11-37.

73 Daborn CJ, Grange JM, Kazwala RR. The bovine tuberculosis cycle - an African perspective. $\mathcal{F}$ Appl Bacteriol (Symposium Supplement) 1996;81:27-32S.

74 Talbot EA, Perkins MD, Silva SFM, et al. Disseminated bacille Calmette-Guérin disease after vaccination: case report and review. Clin Inf Dis 1997;24:1139-46.

75 O'Brien KL, Ruff AJ, Louis MA, et al. Bacillus CalmetteGuerin complications in children born to HIV-1-infected Guerin complications in children born to HIV-1-infected
women with a review of the literature. Pediatrics 1995;95: women

76 Besnard M, Sauvion S, Offredo C, et al. Bacillus CalmetteGuérin infection after vaccination of human immunodeficiency virus-infected children. Pediatr Infect Dis 1993;12: 993-7

77 World Health Organisation. HIV and routine childhood immunization. Wkly Epidemiol Rec 1987;62:297-9.

78 Report. Subcommittee of the Joint Tuberculosis Committee of the British Thoracic Society. Guidelines on the management of tuberculosis and HIV infections in the United Kingdom. BMF 1992;304:1231-3.

\section{Multiple choice questions}

Q1. The following statements are true:

(A) In patients co-infected with tuberculosis and HIV the overall annual risk of developing active tuberculosis rises on average twofold.

(B) In sub-Saharan Africa, there are twice as many men as women infected with HIV.

(C) In Zambia, HIV is the second commonest cause of non-obstetric maternal death.

(D) AIDS patients with active pulmonary tuberculosis predominantly exhibit cavitating granulomatous lung lesions.

(E) Mortality associated with tuberculosis is similar in patients with HIV and in those without HIV.

Q2. In patients disseminated tuberculosis co-infected with HIV:

(A) Tuberculin skin tests may be negative.

(B) Blood cultures for mycobacteria are rarely positive.

(C) Pericarditis is a common extrapulmonary manifestation.

(D) Central nervous system involvement is invariably seen.

(E) Patients are less prone to weight loss than patients with non-HIV associated tuberculosis.

Q3. With regards to treatment for tuberculosis in

HIV infected patients the following are true:

(A) Addition of pyridoxine to antituberculosis regimens prevents ethambutol induced neuropathy.
(B) Extending antituberculosis treatment from six to 12 months results in a lower relapse rate and an improved survival rate.

(C) Rifampicin interacts with antiretrovirals by inhibiting the cytochrome CYP450 enzyme thus reducing the levels of the antiretroviral drugs.

(D) Current recommendations state that antiretrovirals should be continued while a patient is being treated for tuberculosis.

(E) HIV positive patients with tuberculosis experience more hypersensitivity reactions than HIV negative patients with tuberculosis.

Q4. The following statements with regards to tuberculosis are true:

(A) HIV positive patients are more likely to be "smear positive" than HIV negative patients.

(B) Identification of acid fast bacilli on microscopy enables the diagnosis of tuberculosis to be made.

(C) Children with tuberculosis are less likely to be smear positive than adults.

(D) Rifampicin resistance can be reliably predicted using molecular methods to detect mutations in the $R p o B$ gene.

(E) Serology is of use in the diagnosis of tuberculosis in HIV positive patients.

Q5. Which of the statements are true:

(A) Mycobacterium bovis is a recognised cause of primary pulmonary tuberculosis.

(B) Those aged between 5 and 15 years old are at particular risk of tuberculosis.

(C) HIV positive patients, once infected with tuberculosis, are at high risk of reinfection as well as reactivation.

(D) Tuberculosis is expected to cause $30 \%$ of the expected AIDS related deaths in 1999.

(E) In babies infected with HIV it is safe to give $B C G$ vaccination.

Q6. In the year 1999 what percentage of the expected 2.5 million AIDS related deaths are estimated to be due to tuberculosis?

(A) $10 \%$

(B) $30 \%$

(C) $50 \%$

(D) $70 \%$

(E) $90 \%$

Q7. The overall annual risk of developing active tuberculosis infection in persons co-infected with the tubercle bacillus and HIV, compared with persons not infected by HIV, is increased by about:

(A) 10 times

(B) 20 times

(C) 30 times

(D) 40 times

(E) 50 times

Q8. The region currently most affected by HIV and tuberculosis co-infection (the "cursed duet") is:

(A) South East Asia

(B) North Africa

(C) Sub-Saharan Africa

(D) USA

(E) Europe 
Q9. Which ONE of the following statements is false?

(A) The standard WHO recommended antituberculosis treatment is six months with rifampicin, isoniazid, pyrazinamide, and ethambutol/streptomycin.

(B) HIV positive patients are less likely than non-HIV infected patients to complete the course of antituberculosis treatment.

(C) Side effects of antituberculosis treatment are comparatively more common in HIV infected patients with tuberculosis.

(D) Protease inhibitors and rifampicin rarely interact.

(E) Drug resistant tuberculosis is by definition resistant to isoniazid and rifampicin.

\section{Answers to multiple choice questions:}

Q1:

(A) False. The risk is much greater-being in the region of 20 times greater.

(B) False. There is a roughly equal prevalence among the sexes with heterosexual sex being the commonest mode of transmission.

(C) True. The commonest is malaria.

(D) False. HIV infected patients with active pulmonary tuberculosis experience necrotising rather than granulomatous lung lesions that are not walled off by fibrous changes as in tuberculosis in non-HIV patients. This leaves the patient more prone to local invasion and disseminated disease throughout the body (cryptogenic disseminated tuberculosis).

(E) False. Mortality is higher in those with HIV.

Q2:

(A) True. Although the extent of induration is not related to CD4 count.

(B) False. Between one third and one half of cases will be blood culture positive.

(C) True. Pericarditis is the second commonest extrapulmonary manifestation after asymmetrical lymphadenopathy.

(D) False. Central nervous system involvement is actually quite rare in those African patients with HIV and disseminated tuberculosis.

(E) False. Severe wasting in Africans with HIV known as "slim disease" is thought to be associated with tuberculosis.
Q3:

(A) False. Neuropathy is a side effect seen with isoniazid not ethambutol.

(B) False. Survival in HIV positive patients with active tuberculosis is not affected by increasing the length of course of treatment.

(C) False. Rifampicin is an enzyme inducernot inhibitor.

(D) True.

(E) True.

Q4:

(A) False. HIV positive patients with active tuberculosis are less likely to be smear positive.

(B) False. Distinction between tuberculosis and other mycobacterial species (for example, $M$ avium intracellulare) cannot reliably be made on microscopy. The gold standard remains culture of the organism. Molecular methods can also be used.

(C) True.

(D) True.

(E) False. Serology lacks both specificity and sensitivity especially in HIV positive patients.

Q5:

(A) False. $M$ bovis causes primary infection in the pharynx or intestine (via drinking infected milk) rather than the lung. Although isolated institutionalised outbreaks have been reported among HIV infected patients there is no firm evidence that the spread was via primary lung infection.

(B) False. Those within this age group have a relative protection against infection with tuberculosis - the "golden age group".

(C) True.

(D) True.

(E) False. There is an increased incidence of infectious complications with use of this live attenuated vaccine.

Q6:

(B) True 30\% (WHO estimates).

Q7:

(B) True 20 times.

Q8:

(C) True. While many regions are affected, sub-Saharan Africa appears to bear the brunt of the cursed duet of tuberculosis and HIV.

Q9:

(D) True. Rifampicin induces enzymes in the cytochrome $P-450$ system and leads to interactions between these drugs. 